\title{
Biomechanics of thoracic discectomy
}

\author{
Andrew E. Wakefield, M.D., Michael P. Steinmetz, M.D., and EdWard C. Benzel, M.D. \\ Department of Neurosurgery, Hartford Hospital, Hartford, Connecticut; and The Cleveland Clinic \\ Foundation, Department of Neurosurgery, Cleveland, Ohio
}

\begin{abstract}
The thoracic spine is a structurally unique region that renders it uniquely suceptible to thoracic disc herniation. Surgical management strategies are complicated, in part, by the regional anatomical and biomechanical nuances. Surgical approaches include posterior, posterolateral, and anterior routes. Each isassociated with specific indications and contraindications. The biomechanical principles and safe anatomical trajectories must be considered in the surgical decision-making process. These issues are discussed in the pages that follow.
\end{abstract}

KEY WORDS - biomechanics • thoracic spine • instability - discectomy

The thoracic spine has structurally distinct characteristics that distinguish it from the cervical and lumbar spine. A highly specialized region, it is uniquely adapted to an erect posture and the load-bearing demands required from the daily activities of upright creatures. ${ }^{5}$ The thoracic spine has been divided into three regions: 1) the cervicalto-thoracic transition zone (T1-4) 2), the thoracic-to-lumbar transition zone (T10-12), and 3) the midthoracic region (T4-10). ${ }^{16}$ The transition zones are regions exhibiting biomechanical characteristics that are a blend between the adjacent spinal levels.

The kyphotic curve of the thoracic spine appears in early development. Hollingshead described the overall embryological spine as being a C-shaped structure with an anterior concavity. He explained that at birth the cervical spine develops a lordotic posture in response to the paraspinal musculature, and lumbar lordosis is a result of the influence of the psoas muscles. Thus the kyphotic curve of the thoracic spine is a developmental phenomenon, whereas the lordotic curves of the cervical and lumbar spine are adaptive.$^{16}$ In normal sagittal balance the thoracic spine has a natural moment arm that may cause an increase of the kyphotic curve when a disruption of the dorsal tension band and/or loss of the ventral weight-bearing capacity occurs (Fig. 1). Additional structures not present in the cervical or lumbar spine, namely the rib cage and sternum, are present in the thoracic spine. The stabilizing influences of these structures on the thoracic spine are reviewed in this paper. Additionally, the various approaches to the thoracic spine are briefly reviewed in light of their relation to biomechanics so that strategies to determine the extent of postoperative thoracic stability and its management can also be addressed.

Abbreviations used in this paper: $\mathrm{ALL}=$ anterior longitudinal ligament; PLL = posterior longitudinal ligament; ROM = range of motion; VB = vertebral body.

\section{RELEVANT THORACIC ANATOMY}

The posterior thoracic VB height is greater than the anterior height, which explains, in part, the existence of thoracic kyphosis. The size of the thoracic VBs increases as the thoracic spine descends. The facet joints change their orientation from the coronal plane in the cervicothoracic region to a more sagittal plane in the thoracolumbar region, ${ }^{3}$ and this affects spinal motion. There is, for example, limited flexion-extension in the upper thoracic spine, whereas increased flexion-extension is present in the lower thoracic spine. The thoracic pedicles also increase in size as the thoracic spine descends. The pedicles are wide in their sagittal dimension, however, but narrow in their coronal dimension. This phenomenon may complicate the placement of pedicle screws in this region. ${ }^{14}$

The thoracic spine consists of a unique osteoligamentous complex. The costovertebral joints connect the VBs to the rib cage and to adjacent VBs. Articulations of the transverse process with the rib and the rib head to the VB provide spinal stability. The associated ligaments involved with these articulations are the costotransverse, superior costotransverse, radiate, and intraarticular ligaments (Fig. 2 ). This complex provides significant stability compared with the cervical or lumbar spine. The normal ROM in the thoracic spine is 2 to $20^{\circ}$ for flexion-extension, 12 to $18^{\circ}$ for lateral bending, and 4 to $16^{\circ}$ for axial rotation. ${ }^{7}$

\section{BIOMECHANICS OF THE THORACIC SPINE}

Perhaps the best definition of spinal instability was written by White and Panjabi: ${ }^{21}$ "Clinical instability is the loss of the ability of the spine under physiological loads to maintain its pattern of displacement so that there is no initial or additional neurological deficit, no major deformity, and no incapacitating pain." In 1963 Holdsworth ${ }^{11}$ proposed a two-column theory of spinal stability: an anterior column consisting of the VB with its ligamentous struc- 


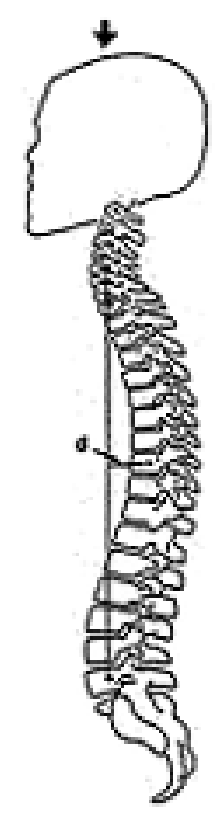

Fig. 1. Drawing showing that the thoracic kyphotic posture increases the length of the natural moment arm (d) and, thus, the magnitude of the bending moment resulting from an eccentrically placed axial load (arrow). Reprinted with permission from Benzel, 1995.

tures and a posterior column consisting of the pedicles and dorsal structures. Although this provided an estimation of instability, it proved to be inadequately comprehensive over time. Twenty years later Denis ${ }^{9}$ introduced a threecolumn model: the anterior column consists of the ALL and the anterior half of the VB; the middle column is composed of the posterior half of the VB and the PLL, and the posterior column is composed of the posterior elements (facet joints and associated ligamentous structures). In another model proposed by Benzel, ${ }^{1}$ the VB is visualized as 27 smaller cubes. This 27 -cube model takes into consideration the integrity of the VB; however, it does not evaluate the posterior elements (Figs. 3 and 4).

The biomechanical properties of the thoracic spine differ from those of the cervical and lumbar spine. In the cervical and lumbar spine, the smallest functional unit con-

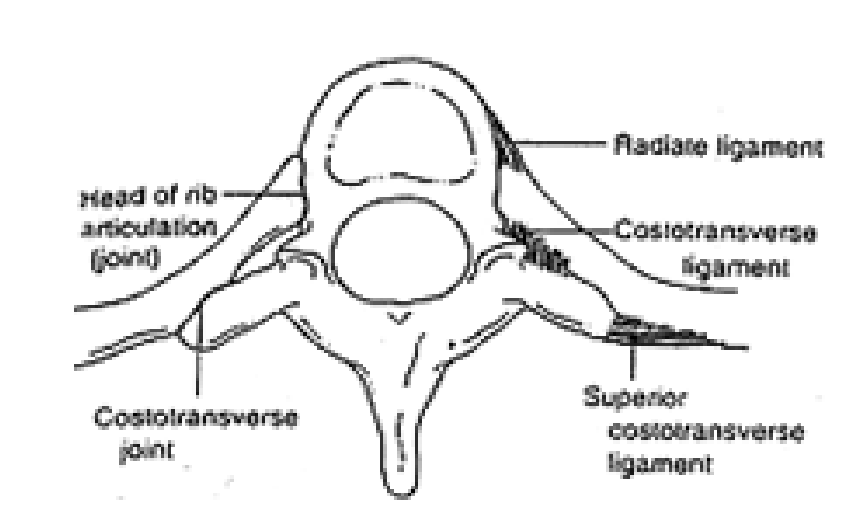

Fig. 2. Drawing showing cross-section through a thoracic vertebra and costovertebral joints. Reprinted with permission from Benzel, 1995.

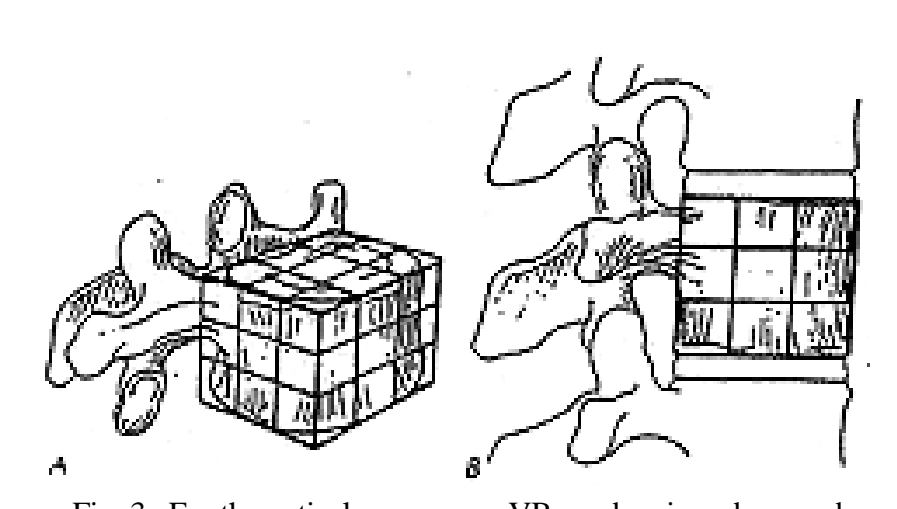

Fig. 3. For theoretical purposes, a VB can be viewed as a cube composed of 27 equal smaller $(3 \times 3 \times 3)$ cubes. A: Oblique view. B: Lateral view. Reprinted with permission from Benzel, 1995.

sists of two VBs and the interconnecting soft tissue. In the thoracic spine, this functional unit is not as straightforward. There are connections between VBs and the rib cage. These consist of the costovertebral and costotransverse joints. Thoracic VBs are connected to adjacent vertebrae via bilateral costovertebral joints. ${ }^{15,20}$

The rib cage is the additional stabilizing component present in the thoracic spine. This sternal-rib complex has been described by Berg ${ }^{6}$ as the fourth column. Oda, et al., ${ }^{15}$ and Tacheuchi, et al., ${ }^{20}$ have performed studies in which they used canine rib cage-thoracic spine complexes. In nondestructive testing, Oda, et al., sectioned the posterior elements at the T-6 and T-7 levels, and they observed an increase in the range of flexion and extension. With sequential sectioning of the costovertebral joints at $\mathrm{T}-7$, a large increase in the neutral zone, lateral bending, and axial rotation resulted. After disruption of the rib cage, further increases in lateral bending and axial rotation were observed. Takeuchi, et al., in addition, concluded that the intervertebral disc regulates the stability of the thoracic spine in flexion-extension, lateral bending, and axial rotation. Reporting a series of experiments in which they used cadaveric torsos, Feiertag, et al., ${ }^{10}$ found that the combination of rib head resection and radical discectomy provided significant increases in thoracic spine motion. A thoracic discectomy (with preservation of the PLL), complete unilateral rib head resection, or a unilateral total facet excision, however, did not cause a significant increase in motion if performed individually. ${ }^{10}$

\section{SURGICAL APPROACHES}

There are basically three approaches to the thoracic spine: 1) posterior, 2) posterolateral, and 3) anterior (Fig. 5). From a biomechanical perspective, many of these have similar characteristics. It should be kept in mind that no single strategy for determining stability of the spine is comprehensive or thorough in its evaluation. The methods of Holdsworth, ${ }^{11}$ Denis, ${ }^{9}$ and Benzel ${ }^{1}$ should be used as guideposts to aid in the thorough process of evaluating spinal stability.

\section{Posterior Approach}

Laminectomy.The initial approach used to treat thoracic disc herniation was a laminectomy. In 1952 Logue $^{14}$ 


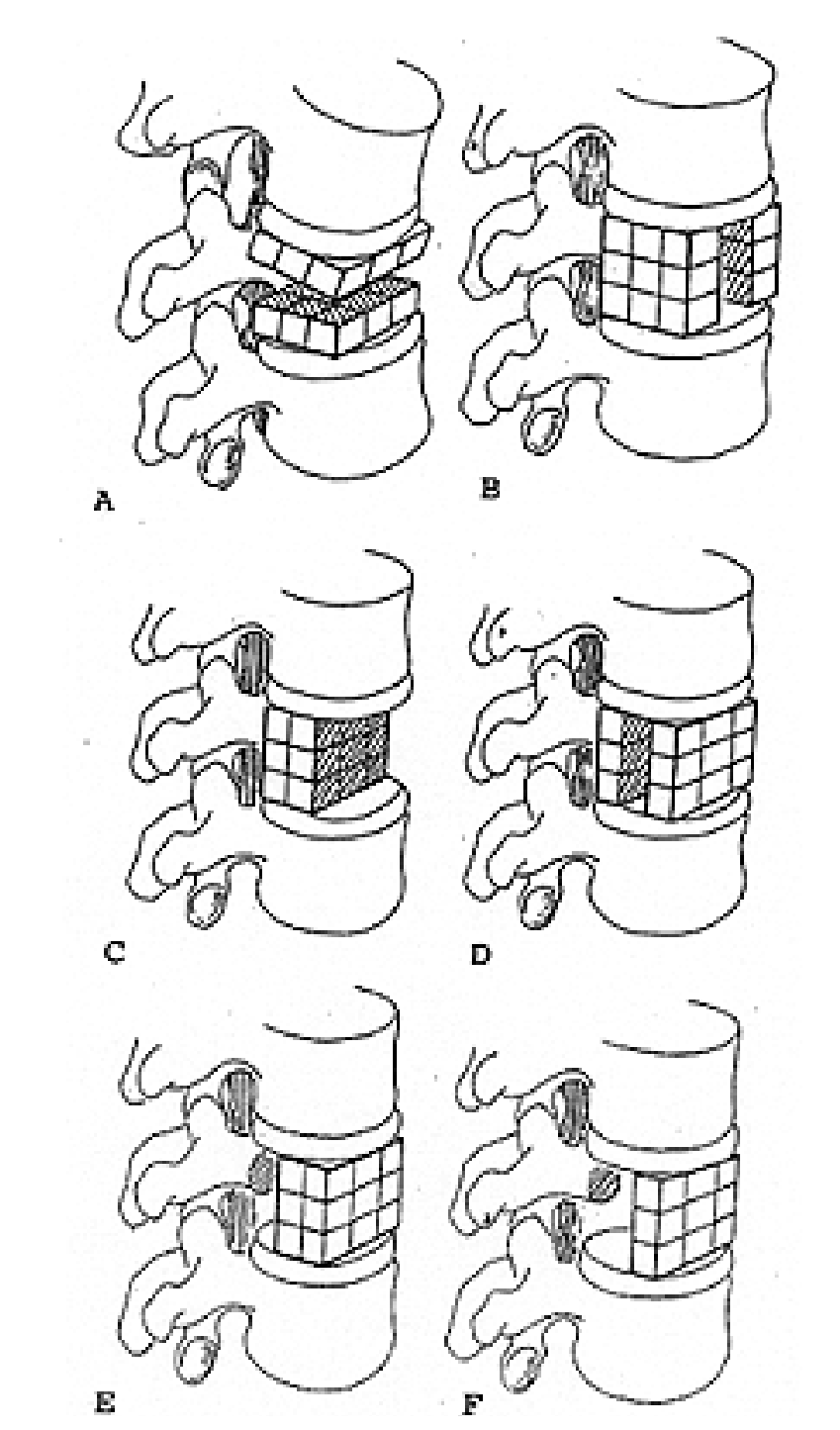

Fig. 4. Drawings showing resections of portions of the "cubic" VB depicted in Fig. 3. A: The resection (or disruption) of the middle axial (horizontal) third of the VB in its sagittal dimension, as might occur following trauma, would create an unstable situation. B: Resection of the middle sagittal (vertical) third of the VB would not significantly destabilize the spine. C-E: Partial vertebrectomy, involving removal of the anterior portion in the coronal plane of the VB (C), affects stability more than resection of the middle (D) or posterior portion (E) of the VB in the coronal plane. This assumes that the posterior columns are intact. F: In fact, resection of the posterior and middle portions of the VB may not significantly disrupt spinal integrity with intact posterior columns. Reprinted with permission from Benzel, 1995.

reported that the results were poor and a significant number of the patients were left paraplegic after using this approach. ${ }^{4}$ Thus, laminectomy is not recommended for thoracic disc herniation, although it may still be used for thoracic spondylosis. Whereas the posterior tension band is adversely affected, the remaining structures are left intact (that is, the anterior and middle columns in Denis' three-column model $^{9}$ and the 27-cubes in Benzel's model ${ }^{1}$ are undisturbed (Fig. 5A). The fourth column (sternal-rib complex) of Berg is also not disrupted. Therefore, signif-

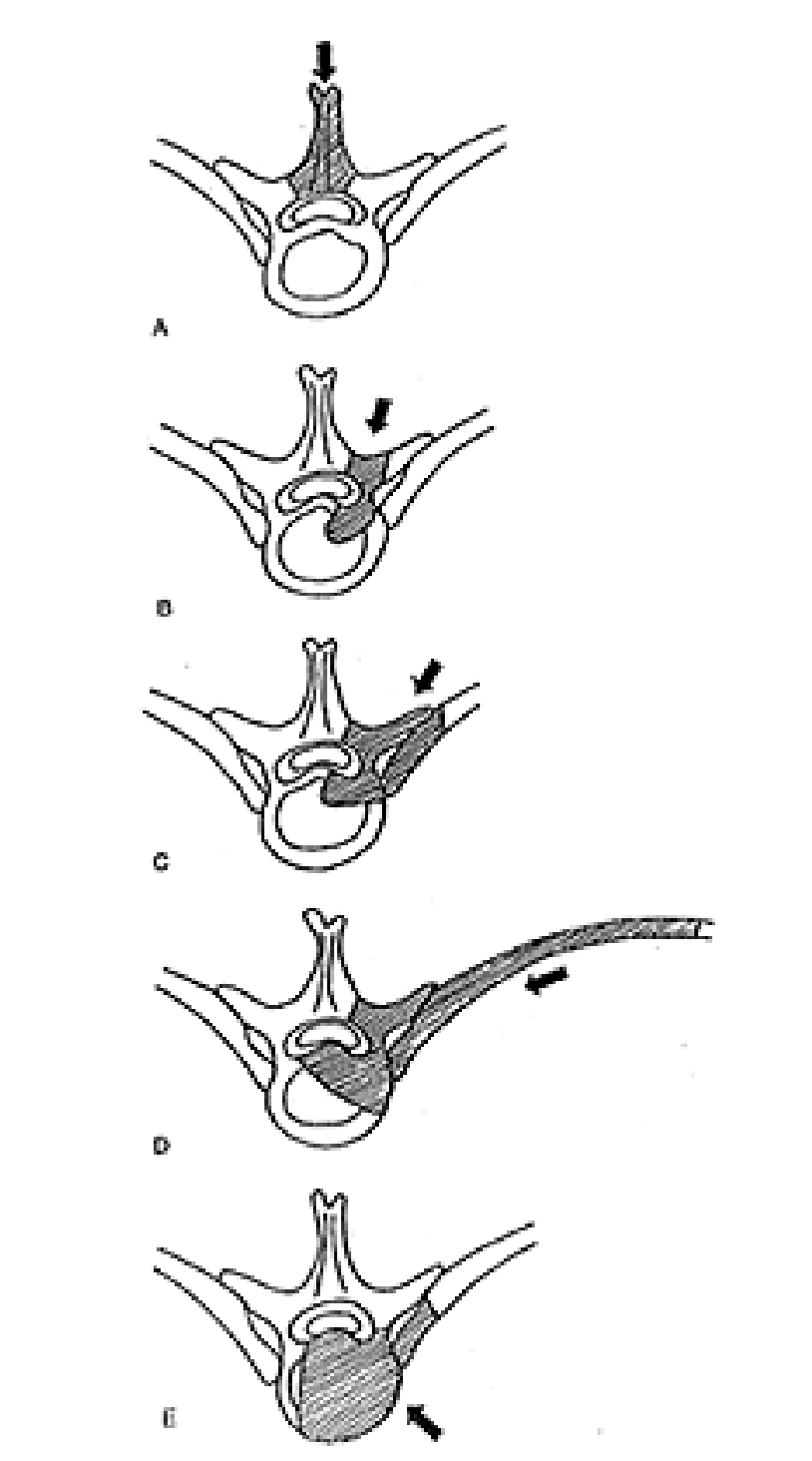

Fig. 5. Drawings illustrating alternative approaches to the thoracic spine: laminectomy (A); transpedicular approach (B); costrotransversectomy (C); lateral extracavitary approach (D); and anterior intracavitary approach $(\mathrm{E})$. Reprinted with permission from Benzel, 1995. Arrows depict trajectory of surgery.

icant spinal instability should be uncommon when this surgical approach is used.

Transpedicular.This approach was described in 1978 by Patterson and Arbit. ${ }^{19}$ The structures affected by this approach are the facet joint, the pedicle caudal to the disc space which is drilled flush with the VB, and the VB itself. The extent of VB removal usually only involves a cross-sectional area loss of 1.5 to $2 \mathrm{~cm}$ and is limited to the lateral aspect of the VB (Fig. 5B). Structures affected by this approach are the facet joint, the pedicle, and the posterolateral aspect of the disc. Depending on the extent of the laminectomy performed, the posterior tension band may be preserved. The unilateral resection of the facet joint, pedicle and the postero-lateral removal of the annulus fibrosus and disc should not destabilize the spine. Takeuchi, et al., ${ }^{20}$ demonstrated an increase in flexionextension, lateral bending, and axial rotation following 
thoracic discectomy in a canine model. They performed an anterior approach, however, with disruption of the ALL. As with laminectomy, the fourth column of Berg is preserved when using this approach, and significant support for the thoracic spine remains. Patients, however, have reported localized back pain postoperatively. Removal of the pedicle has been implicated as the cause of this pain. ${ }^{12}$

Transfacet Pedicle Sparing. Stillerman, et al., ${ }^{19}$ described the transfacet pedicle-sparing approach in 1995. The pedicle is spared, as the term implies. Exposure is obtained via a keyhole facetectomy. Therefore, only a partial facetectomy is performed. The facet-pedicle complex is preserved with this technique, which has been shown to improve outcome relating to axial pain. ${ }^{19}$ Additionally, there is minimal disruption of anterior and posterior spinal elements so that spinal instability is uncommon.

\section{Lateral Approaches}

The Lateral Extracavitary Approach.This approach was developed by Capener and refined by Larson, et al. ${ }^{13}$ The proximal 8 to $12 \mathrm{~cm}$ of rib is resected. The pedicle caudal to the desired disc space is partially or completely removed (Fig. 5D). The disc space is evacuated and the PLL is taken down. The dorsocaudal quarter of the rostral vertebra and the dorsorostral quarter of the caudal vertebra are drilled out. The costotransverse joints are removed. This provides access for discectomy and, if appropriate, bone stock fusion.

Costrotransversectomy. Menard initially described the costrotransversectomy in 1894 for the treatment of Pott disease. $^{2}$ A costotransversectomy is similar to the lateral extracavitary approach, but more of a posterior approach is required (Fig. 5C). Essentially, the same procedure is performed as with the lateral extracavitary approach but with a more limited rib resection. Also of note is that the lateral extracavitary approach allows the surgeon to access the VB from beneath the erector spinae muscle, whereas the costotransversectomy approach provides access to the $\mathrm{VB}$ via a route medial to this muscle.

The authors of studies performed in animal and human cadaver models have shown that there is a significant increase in the ROM in all planes after resection of the rib, costovertebral and costotransverse joints, pedical, PLL, and the annulus fibrosus and disc. ${ }^{10,15,20}$ In Benzel's 27cube model ${ }^{1}$ sufficient anterior support is usually present. If the posterior tension band or the sternal-rib complex that support the spine are disrupted, however, fusion is warranted. If the anterior column requires reconstruction, the resected rib may be used for fusion.

\section{Anterior and Anterolateral Approaches}

Transthoracic Thoracotomy. This approach was described by Craaford, et al., ${ }^{8}$ in 1958 and was later refined by Ransohoff, et al., ${ }^{18}$ and Perot and Munro ${ }^{17}$ in 1969. The transthoracic approach provides excellent exposure of the anterolateral thoracic spine and is particularly useful for centrally located calcified disc herniations or for multilevel exposure (Fig. 5E). A rib is partially resected, usually at the level above the disc space, and a thoracotomy is performed. This approach involves resection of the caudal pedicle (optional), the costovertebral joint, the anterolateral annulus fibrosus and disc, and the PLL. The rostral and caudal VBs are removed, to a varying degree, for exposure. Because the posterior elements are not disrupted, the posterior tension band is preserved. Similar to the lateral extracavitary and the costotransversectomy approaches, however, the middle and anterior columns are interrupted, as is the anterior load-bearing capability. Many authors have advocated fusion because of the risk of delayed kyphosis. Benzel's 27-cube model ${ }^{1}$ is useful to determine the anterior load-bearing capacity under these circumstances (Fig. 6).

Transsternal Thoracotomy. In this approach, the sternum is split and the upper thoracic spine approached anteriorally. The working space is between the left common carotid artery and the brachiocephalic artery, the trachea, esophagus, and thyroid gland. This approach is truly anterior. The ALL, annulus fibrosus, and disc are disrupted. The PLL is also usually removed. Takeuchi, et al., ${ }^{20}$ using a canine model, demonstrated a significant increase in the ROM following anterior discectomy. The ROM was further increased after resection of the rib head. In this approach, the rib head is not resected, but the integrity of the sternal-rib complex is violated because of the splitting of the sternum. Placement of a bone graft is warranted to re-

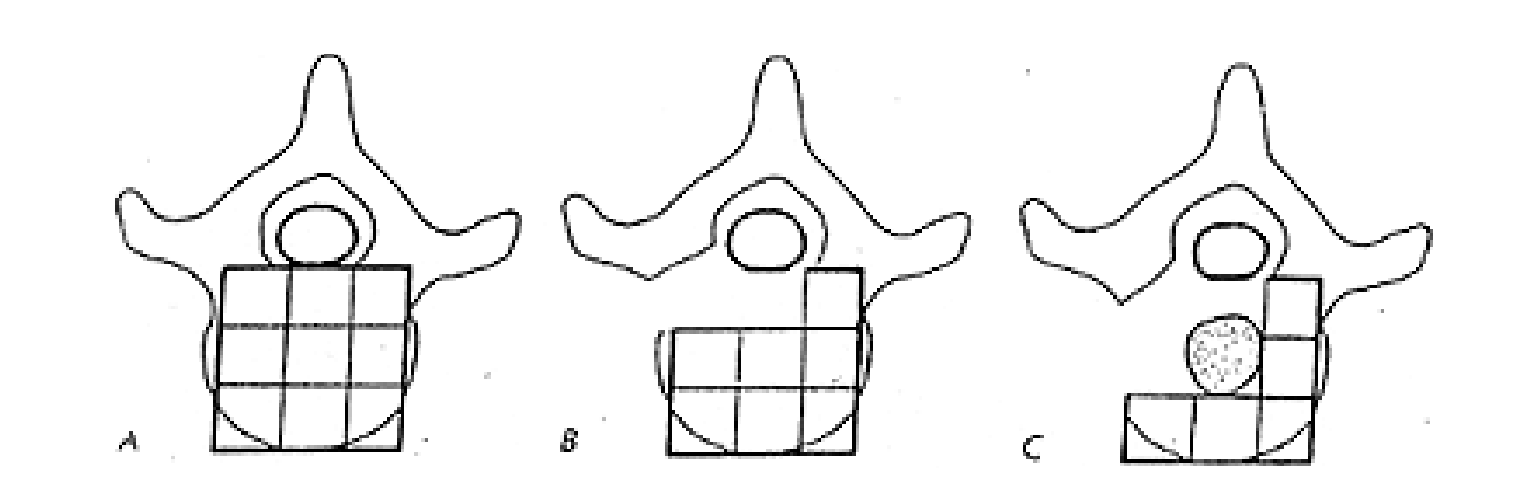

Fig. 6. The 27-cube model can be used to depict the bone resection accomplished via a lateral extracavitary decompression of the thoracic dural sac. A: Preoperative view of the spine. B: Resection of bone limited to the posterior aspect of the VB allows for decompression while preserving bone integrity and weight bearing. Further osseous resection is then required for strut graft placement. C: The final extent of bone removal, with the bone graft (stippled area) in place. Reprinted with permission from Benzel, 1995. 
establish the integrity of the anterior and middle columns to prevent delayed kyphosis.

\section{CONCLUSION}

The thoracic spine has unique anatomical and biomechanical properties that are distinct from the cervical and lumbar spine. Several factors must be evaluated when determining the stability of the thoracic spine. The kyphotic curvature yields a natural-occurring moment arm anterior to the spine. Forces are therefore perpetually present that tend to increase the kyphosis if the anterior column is not preserved or is weakened because of surgery. The sternal-rib complex (the fourth column) provides significant support for the thoracic spine. Even unilateral disruption can cause an increase in the ROM in all planes. Finally, the integrity of the vertebral column must be figured into the evaluation. The quality of the bone, the kyphotic angle, and whether a scoliotic curve is present have an impact on the stability of the spine following discectomy. As stated previously, no single method of evaluating spinal stability is comprehensive or complete, and like many aspects of spine surgery, it is the surgeon's experience and judgment that ultimately determine.

\section{References}

1. Benzel EC: Biomechanics of Spine Stabilization: Principles and Clinical Practice. New York: McGraw-Hill, 1995, pp 97-102

2. Benzel EC: Spine Surgery: Techniques, Complication Avoidance, and Management. New York: Churchill Livingstone, 1999, pp 1-21

3. Benzel EC: Spine Surgery: Techniques, Complication Avoidance, and Management. New York: Churchill Livingstone, 1999, pp 93-118

4. Benzel EC: Spine Surgery: Techniques, Complication Avoidance, and Management. New York: Churchill Livingstone, 1999, pp 369-387

5. Benzel EC, Stillerman CB: The Thoracic Spine. St Louis: Quality Medical Publishing, 1999, pp 68-79

6. Berg EE: The sternal-rib complex. A possible fourth column in thoracic spine fractures. Spine 18:1916-1919, 1993

7. Broc GG, Crawford NR, Sonntag VKH, et al: Biomechanical effects of transthoracic microdiscectomy. Spine 22:605-612, 1997

8. Crafoord C, Heirtonn T, Lindblom K, et al: Spinal cord com- pression caused by a protruded thoracic disc: report of a case treated with anterio-lateral fenestration of the disc. Acta Orthop Scand 28: 103-107, 1958

9. Denis F: The three column spine and its significance in the classification of acute thoracolumbar spinal injuries. Spine 8: 817-831, 1983

10. Feiertag MA, Horton WC, Norman JT, et al: The effect of different surgical releases on thoracic spinal motion. A cadaveric study. Spine 20:1604-1611, 1995

11. Holdsworth F: Fractures, dislocations, and fracture-dislocations of the spine. J Bone Joint Surg Br 45:6-20, 1963

12. Kothe R, Panjabi MM, Liu W: Multidirectional instability of the thoracic spine due to iatrogenic pedicle injuries during transpedicular fixation. A biomechanical investigation. Spine 22: 1836-1842, 1997

13. Larson SJ, Holst RA, Hemmy DC, et al: Lateral extracavitary approach to traumatic lesions of the thoracic and lumbar spine. J Neurosurg 45:628-637, 1976

14. Logue V: Thoracic intervertebral disc prolapse with spinal cord compression. J Neurol Neurosurg Psychiatry 15:227-241, 1952

15. Oda I, Abumi K, Lu D, et al: Biomechanical role of the posterior elements, costovertebral joints, and rib cage in the stability of the thoracic spine. Spine 21:1423-1429, 1996

16. Panjabi MM, Takata K, Goel V, et al: Thoracic human vertebrae. Quantitative three-dimensional anatomy. Spine 16: 888-901, 1991

17. Perot PL Jr, Munro DD: Transthoracic removal of midline thoracic disc protrusions causing spinal cord compression. J Neurosurg 31:452-458, 1969

18. Ransohoff J, Spencer F, Siew F, et al: Transthoracic removal of thoracic disc. Report of three cases. J Neurosurg 31:459-461, 1969

19. Stillerman CB, Chen TC, Day JD, et al: The transfacet pediclesparing approach for thoracic disc removal: cadaveric morphometric analysis and preliminary clinical experience. J Neurosurg 83:971-976, 1995

20. Takeuchi T, Abumi K, Shono Y, et al: Biomechanical role of the intervertebral disc and costovertebral joint in stability of the thoracic spine. A canine model study. Spine 24:1414-1420, 1999

21. White AA, Panjabi MM: Clinical Biomechanics of the Spine, ed 2. Philadelphia: JB Lippincott, 1990, pp 278-378

Manuscript received July 12, 2001.

Accepted in final form August 16, 2001.

Address reprint requests to: Edward Benzel, M.D., Department of Neurosurgery, The Cleveland Clinic Foundation, 9500 Euclid Avenue, S80, Cleveland, Ohio 44195. email:benzele@ccf.org. 